\title{
Impact of magnetic field on beta-processes in partially transparent medium
}

\author{
Alexandra Dobrynina* \\ P. G. Demidov Yaroslavl State University, Yaroslavl, Russia \\ E-mail: dobrynina@uniayr.ac.ru \\ Igor Ognev \\ P. G. Demidov Yaroslavl State University, Yaroslavl, Russia \\ E-mail: ognev@uniyar.ac.ru
}

\begin{abstract}
An influence of a magnetic field on beta-processes is investigated in a partially transparent for neutrinos medium of a core-collapse supernova. It is shown that significant modifications of these reactions are only possible in the supernovae with extremely strong magnetic fields. This means that many problems, like a neutrino propagation in such objects, can be solved without accounting of the magnetic field, even within magnetorotational models, where a strong magnetic field is generated naturally.
\end{abstract}

European Physical Society Conference on High Energy Physics - EPS-HEP2019 10-17 July, 2019

Ghent, Belgium

${ }^{*}$ Speaker. 


\section{Introduction}

A supernova explosion with a collapse of its central part is one of unresolved problems of modern astrophysics [1]. In simulations, a successful explosion can occur due to various factors, and a neutrino interaction with a supernova matter is an important component of all existing models. A great progress has been achieved in magnetorotational models, in which the explosion occurs due to a strong-magnetic-field generation in the supernova. Indirectly, the presence of a strong magnetic field in supernovae is confirmed by observations of neutron stars with the magnetic field strengths up to $10^{15} \mathrm{G}$ [2], known as magnetars. So, it is important to study an interaction of neutrino fluxes with a supernova or magnetar matter in the presence of a strong magnetic field background and selected numerical results for the supernova are presented in this proceedings.

\section{Results}

In this paper we study the magnetic-field influence on $\beta$-processes in a supernova matter which is partially transparent for neutrinos. In such an environment, $\beta$-decay and its inverse process are kinematically suppressed and the main reactions are $p+e^{-} \leftrightarrow n+v_{e}$ and $n+e^{+} \leftrightarrow p+\bar{v}_{e}$.

Calculations are based on an emissivity of a matter presented in Ref. [3]. Note that the result used is valid for an arbitrary magnetic field strength (it is restricted from above by the requirement of an existence of the spontaneous symmetry breaking in the Standard Model). Because the neutrino interaction with the matter significantly affects dynamics in highly-heating regions inside a supernova, an electron-positron plasma existing in them is assumed to be ultrarelativistic. In addition, we suppose that nucleons are non-degenerate and electron-positron plasma is moderately degenerate. These conditions are well satisfied outside the inner part of the supernova. It should be noted that in the inner part neutrinos are not free but are in the thermodynamic equilibrium with the matter. We also assume that the neutrino propagation is spherically symmetric. We estimate a reaction rate $\Gamma$ (the number of processes occurring in a unit volume per unit time) as well as energy $Q$ and three-momentum projection $\mathscr{F}_{r}$ on the radial direction, transferred from (anti)neutrinos to a unit volume of the medium per unit time.

These quantities depend on a large number of parameters relevant for both the matter and neutrino radiation. A complete analysis of the magnetic field influence on them is a difficult and very ambiguous problem. Likely, it is possible to fix some parameters by using the data provided by numerical simulations. In our analysis we use results of the SN 1D simulations performed with the PROMETHEUS-VERTEX code developed by H.-T. Janka and his collaborators [4] ${ }^{1}$. In this analysis the SN progenitor mass is equal to $27 M_{\odot}$ and the resultant neutron star has the baryonic mass $1.76 M_{\odot}$. Based on the data available, we were able to fix all the parameters except two: the distance $R$ from the neutron-star center and the time $t$ after a bounce. Additional parameters in our problem are the magnetic field strength and its spatial configuration. To be definite, in our numerical analysis we use the magnetic-field configuration with $B_{r}=0$ being spatially homogeneous. Note that the main results obtained are the same for other magnetic-field configurations as well.

To estimate an importance of the magnetic field for beta-processes under supernova conditions, it is convenient to find a relative deviation of the macroscopic quantities specified above, which are

\footnotetext{
${ }^{1}$ https://wwwmpa.mpa-garching.mpg.de/ccsnarchive/archive.html
} 

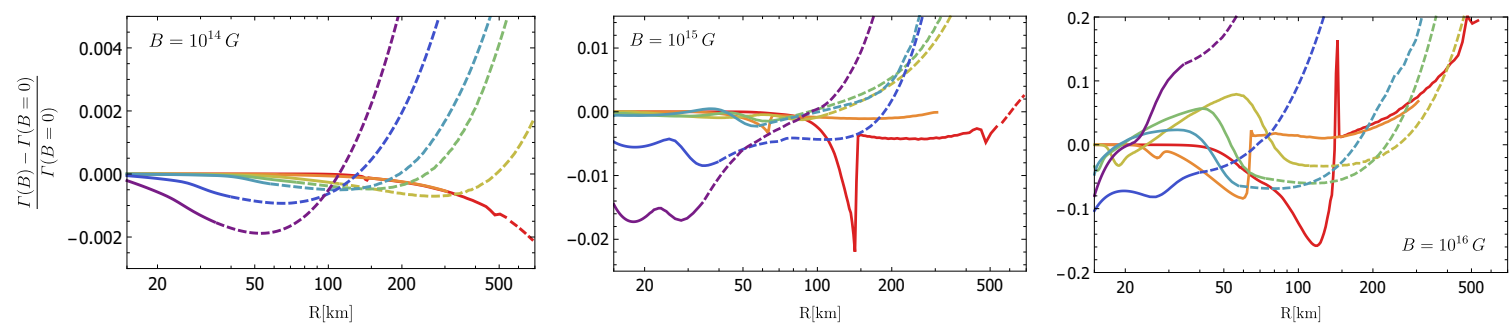

Figure 1: The deviation of the total rate of the proton-to-neutron transition, calculated in the magnetic field, relative to the unmagnetized case in dependence on the distance $R$ from the neutron-star center for fixed values of time and the magnetic field strength. The field strength is explicitly shown in each plot. Red lines: $t=0.1 \mathrm{sec}$; orange: $t=0.5 \mathrm{sec}$; yellow: $t=1.5 \mathrm{sec} ;$ green: $t=4 \mathrm{sec} ;$ cyan: $t=5.5 \mathrm{sec} ;$ blue: $t=10 \mathrm{sec}$; violet: $t=13$ sec.

calculated in the the magnetic field, from the same without the field. The relative deviations of the total rate $\Gamma$ for the proton-to-neutron transition in dependence on the distance $R$ in $\mathrm{km}$ for several fixed values of magnetic field and time are presented in Fig. 1. The dashed parts of the lines describe the supernova regions where the electron-positron plasma is no longer ultrarelativistic, and one should perform corresponding calculations differently. As seen in Fig. 1, the magnetic field significantly affects the reaction rates of the processes in the low-temperature region, where the ultrarelativistic property of plasma is questionable. In addition, the influence of the magnetic field becomes more pronounced with a time evolution, reflecting the stages of supernova cooling. The other important conclusion is that the magnetic field with the strength $B \sim 10^{15} \mathrm{G}$ modifies the rates of beta-processes on a few percents only. Unfortunately, such a magnetic field is difficult to reach in supernova simulations at present.
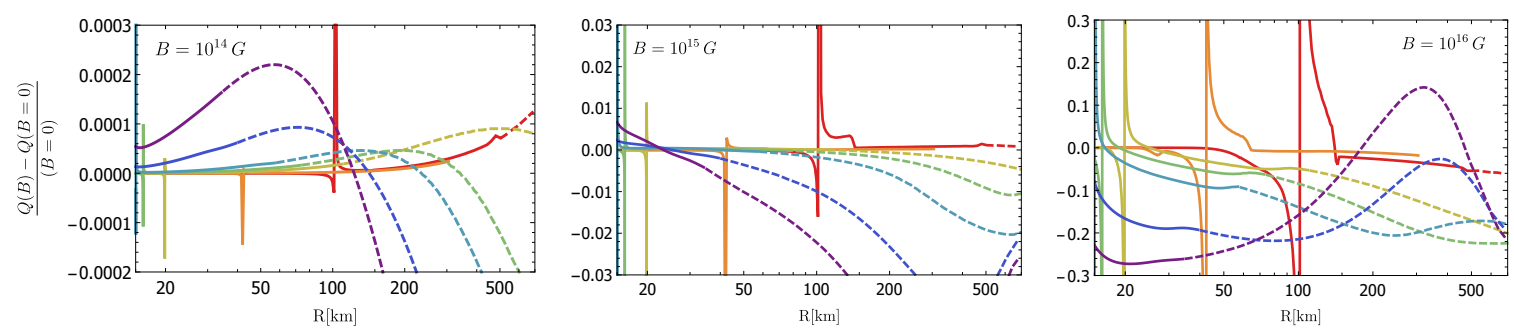

Figure 2: The relative deviation of the total energy transferred from (anti)neutrinos to the matter. The legend is the same as in Fig. 1.

We present similar plots for the heating rate of $\beta$-processes in Fig. 2. The spikes are related with the gain radius, where the total energy $Q$ goes through zero and changes its sign. As seen, the character of the magnetic field influence on the matter heating has similarities with the reaction rates.

The relative deviation $\left(\mathscr{F}_{r}(B) / \mathscr{F}_{r}(B=0)-1\right)$ of the radial projection of the total threemomentum, transferred from (anti)neutrinos to the matter, in the magnetic field background from the unmagnetized one for fixed values of the magnetic-field strength and at different times is presented in Fig. 3. When the functions are negative, this component of the momentum is suppressed 

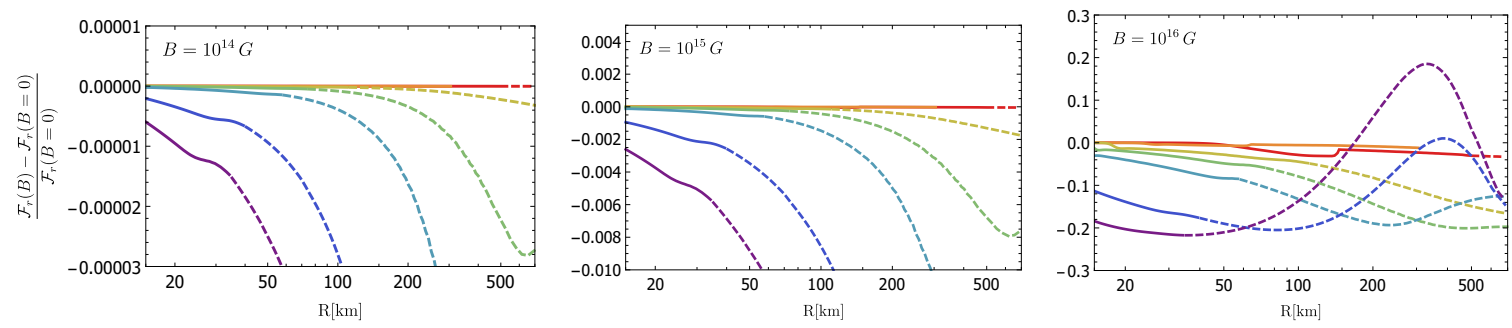

Figure 3: The relative deviation of the radial projection of the total three-momentum transferred from (anti)neutrinos to the matter. The legend is the same as in Fig. 1.

by the magnetic field. In general, this could be bad for the explosion which requires the matter heating but numerically this effect is small and practically irrelevant in realistic supernova conditions. Finally, when the magnetic field value increases, the relative deviation increases too.

\section{Conclusions}

An influence of a magnetic field on beta-processes is investigated in a partially transparent for neutrinos matter outside of the supernova inner part. The magnetic field influence on decay rates, energy and momentum transferred from the neutrinos to the supernova matter is relatively small. Modifications of these quantities on a few percents can be reached when the magnetic field is strong, up to $10^{15} \mathrm{G}$. At present, such a magnetic field is difficult to reach in supernova simulations. Therefore, in many problems related to a supernova explosion, the magnetic field influence can be safely ignored, at least the field effects connecting with the neutrino propagation and processes with the neutrino participation.

\section{Acknowledgments}

The work is supported by the Russian Science Foundation (Grant No. 18-72-10070). We are thankful to H.-T. Janka and his collaborators for providing us with their data of the supernova explosion and for helpful discussions. We are grateful to A. Parkhomenho and G. Raffelt for critical remarks.

\section{References}

[1] A. W. Alsabti and P. Murdin, Handbook of Supernovae. Springer, 2017.

[2] S. A. Olausen and V. M. Kaspi, The McGill Magnetar Catalog, ApJS 212, 6 (2014).

[3] I. S. Ognev, Effect of magnetic field on beta processes in a relativistic moderately degenerate plasma, JETP 123, no. 4, 643 (2016).

[4] L. Hüdepohl, Neutrinos from the formation, cooling, and black hole collapse of neutron stars, $\mathrm{PhD}$ thesis, Technische Universität München, 2014. 